\title{
EP-58
}

\section{How to reduce biliary complication in living donor liver transplantation?}

\author{
Eun-Kyoung JWA*, Joo Dong KIM, Dong Lak CHOI
}

Division of Hepatobiliary Pancreas Surgery and Liver Transplantation, Daegu Catholic University School of Medicine, Daegu, Korea

Introduction: Biliary complication is Achilles in LDLT. Many studies have studied risk factors associated with biliary tract complications. We review our two year LDLT data and find ways to reduce the biliary complications.

Methods: Institutional LT database was searched from 2015.01.01 to 2016.12.31. Their medical records and imaging studies were reviewed.

Results: Between 2015-2016 we did 126 Liver transplantation. (LDLT 106, DDLT 20) In LDLT, total 19 patient suffered from biliary complication. (17.9\%) Two patients suffered from bile leak and 16 patients suffered form biliary stricture and 1 patient suffered from both leakage and stricture. Almost Among the patients who suffered from biliary stricture only one patient treat ERCP the others treated with PTBD insertion. In biliary complication group's 5-year graft survival was 78.9\% which was lower than non biliary complication group (83.9\%) but no significant difference. Compared to the biliary complication group and non biliary complication groups, only $\mathrm{ABO}$ incomplete factor was significant risk factor. Interestingly, duct anastomosis, stent types, duct size were not significant risk factors.

Conclusions: Still biliary complication incidence is common complication in LDLT. There was no significant differences between stent types, duct anastomosis method, duct size. We should try to reduce biliary complication. we could design a prospective study to reduce biliary complications. 